\title{
Profile of cases suspected of attempted suicide by exogenous intoxication
}

\author{
Fernanda Pamela Machado, Marcos Hirata Soares, Patricia Dias Francisquini, Layla Karina Ferrari Ramos \\ Mental Health Research and Study Group, Universidade Estadual de londrina, Londrina, Paraná, Brazil
}

Received: May 16, 2018

Accepted: October 14, $2018 \quad$ Online Published: October 26, 2018

DOI: $10.5430 /$ jnep.v9n2p123

URL: https://doi.org/10.5430/jnep.v9n2p123

\begin{abstract}
Objective: To characterize the profile of patients suspected of attempting suicide, based on cases reported by the Toxicological Information Center of the University hospital of Southern Brazil.

Methods: This is a retrospective aggregate study, based on the analysis of medical records and files between January 2009 and December 2012, aiming to collect information that would create the profile of patients suspected of attempting suicide, from the cases notified by the Toxicological Information Center.

Results: Regarding the physical consequences and the danger of the suicide attempt, $97 \%$ of the suicide attempts did not present life threatening $(n=1,605) ; 85.4 \%$ were not referred to any professional service $(n=1,412)$. The year 2012 was the year with the highest number of suicide attempts that the other years.

Conclusions: It is considered important to improve the notification form for poisoning injuries, to better detect information about suicide attempts.
\end{abstract}

Key Words: Suicide, Poisoning, Psychiatric nursing, Mental health

\section{INTRODUCTION}

Using epidemiological data from the World Health Organization (WHO). ${ }^{[1]}$ It is possible to understand how suicide has become a public health problem. For in 2014, in the world, about 800 thousand people died of suicide. The age group between 15 and 29 years old and the elderly over 70 years old are among the people most affected by this cause of death in both men and women in different regions of the world. ${ }^{[1]}$

When calculated the total and the ratio between the suicide rate of men and women in developing countries is 1.6, while in developed countries 3.5. In general, suicide attempts are more commonly reported by women, but men are more successful. ${ }^{[1]}$

The Brazil, despite having a lower rate, is one of the ten countries that record the highest number of suicides. ${ }^{[1]}$ Because it deals with a topic that is rarely commented on in some countries, $\mathrm{WHO}^{[1]}$ considers that the number is underestimated 20 times, due to lack/absence of records of occurrences or underreporting, because each suicide confirmed, people close to the suicide will have their lives compromised emotionally, economically and socially.

Suicide has been considered a public health problem, mainly affecting young and old of both sexes. It is estimated that by 2020 the number of suicides increases to 1.6 million, that is, one death every 40 seconds. ${ }^{[1]}$ Ingestion of pesticides, suspension and firearms are among the most common suicide methods globally, but many other methods are used as a choice.

One of the methods used is exogenous poisoning by insecticides, carbamates and organophosphates, considered

\footnotetext{
*Correspondence: Fernanda Pamela Machado; Email: Fer.machado3@ hotmail.com; Address: Mental Health Research and Study Group, Universidade Estadual de londrina, Londrina, Paraná, Brazil.
} 
a frequent problem in the emergency services of large hospitals, whether accidentally ingested in children or attempted suicide. ${ }^{[2]}$ Exogenous intoxication may be understood as the clinical and/or biochemical consequence of exposure to chemicals found in the environment or isolated. As an example of environmental intoxicants, one can cite air-to-water foods, plants, venomous or venomous animals. In turn, the main representatives of isolated substances are pesticides, medicines, industrial or household chemicals.

Research investigating the method of choice for suicide reported the use of drugs and pesticides as the most frequently chosen by female adolescentes. ${ }^{[3]}$ For some authors, ${ }^{[4]} 62.5 \%$ used drugs, while another study, 39.6\% used drugs and 33.3\% pesticides. ${ }^{[5]}$

Thus, it is believed that it is of great relevance to characterize the profile of patients with suspected suicide attempt, reported by the Toxicological Information Center (TIC) of a public university reference hospital in the north of the State of Paraná.

\section{METHOD}

This is a retrospective, time-series study based on the analysis of medical files and files between January 2009 and December 2012, aiming to collect information from patients suspected of attempting suicide, from the cases notified by the TIC, as to the origin, destination, care provided, diagnostic classification of the suicide attempt, care received and the consequences of attempted health.

The data collection instrument was based on the protocol Multisite Intervention Study on Suicidal Behaviors (SUPREMISS) developed by the WHO, in a multicenter study in Brazil, China, Estonia, India, Iran, South Africa, Sri Lanka and Vietnam for research on suicide. ${ }^{[6]}$

\subsection{Inclusion criteria}

Records in medical records notified by the IT for exogenous intoxication investigation, considered suspected of suicide attempts.

\subsection{Exclusion criteria}

Patients who received care at TIC due to other type of poisoning, other than exogenous.

\subsection{Data collection}

Data were collected in the TIC, which is inserted into the north of Paraná University Hospital (HURNEP), from the system of chips for Notifiable Diseases Information System and records, when there was the need to complement the information. The purpose of is to record and process data on reporting diseases throughout the country, providing information for analyzing the morbidity profile and thus contributing to decision making at the municipal, state and federal levels. The research was approved by the Committee of Ethics in Research, CAAE n. 0289.0.268.000-11.

\subsection{Data analysis}

The data were analyzed in the Statistical Package for Social Sciences (SPSS) version 21. The Kolmogorov-Smirnov and Shapiro-Wilk tests were used to test the sample normality, indicating that for all the variables tested, the sample distribution is not normal $(p<.001)$. Thus, the Spearman Correlation was used, among the variables, time of hospitalization, consequence of the attempt and type of care received.

\section{RESUltS}

The majority of patients who underwent care came from other "Cities", 1,041 cases (62.9\%) and "Other states" 39 cases $(2.4 \%)$, totaling 1,654 patients.

The variable's suicide attempts was distributed quarterly in the year 2009 to 2012. The lowest incidence was in the quarter of July/August/September $2010(n=75)$ and the highest $(n=162$ ) occurred in the period from January to March 2012. In 2010, the number of suicide attempts remained similar to 2009 ( $n=338$ ), but in 2011 and 2012, there was a significant increase. In $2011(n=530)$ and in $2012(n=549)$. In the year 2012, in the months between October and December, towards the end of the year, suicide attempts have a higher rate than in the other months $(n=138)$.

Table 1. Sociodemographic characteristics of patients treated by CIT for suicide attempt, 2009 to 2012

\begin{tabular}{lll}
\hline \multirow{2}{*}{ Variable } & $\mathbf{n}$ & $\mathbf{\%}$ \\
\cline { 2 - 3 } & $\mathbf{1 , 6 5 4}$ & $\mathbf{1 0 0 . 0}$ \\
\hline Sex & 682 & 41.2 \\
Women's & 972 & 58.8 \\
Men's & & \\
Level of schooling & 885 & 53,5 \\
Ignored & 242 & 14.6 \\
Incomplete high school & 225 & 13.6 \\
Incomplete elementary school & 114 & 6.9 \\
Complete high school & 84 & 5.1 \\
Complete primary education & 53 & 3.2 \\
Incomplete upper level & 51 & 3.1 \\
Higher level & & \\
Zona de moradia & 1,517 & 91.7 \\
Urban area & 137 & 8.3 \\
Rural zone &
\end{tabular}

The mean number of years lived was 30 years and the age range ranged from 17 to 75 years, presenting a standard deviation of \pm 3.8 . The percentage of suicide attempts was $58.8 \%(n=972)$ for males and $42.1 \%(n=682)$ for females. 
The level of schooling ignored prevailed, representing $53.5 \%$ $(\mathrm{n}=885)$, leading to loss of important information. It should be noted that patients with incomplete levels of schooling presented the highest number of suicide attempts, when compared to the complete upper level. It was more frequent among patients with incomplete secondary education, $14.6 \%$ $(n=242)$, followed by incomplete elementary school $13.6 \%$ $(n=225)$. The variable that presented the lowest number of suicide attempts was $3.1 \%(n=51)$, followed by the incomplete upper level (3.2\%). After the suicide attempt, most of the patients attended by required hospital care (see Tables 1 and 2).

Regarding the place of care, the patients who were attended by the emergency department, followed by hospital discharge, represent $60.3 \%(n=998) ; 36.4 \%(n=602)$ remained on observation, received emergency treatment and then were discharged from hospital. Only 3.3\% $(n=4)$ of the patients received transfers to the intensive care unit, other clinics or surgical/ward units.

Regarding the risk of death, $2.7 \%(n=45)$ presented some life threatening. Of the cases identified in urgent care, $0.2 \%$ $(\mathrm{n}=4)$ did not require any treatment. In relation to hospitalization time, $60.08 \%(\mathrm{n}=1,005)$ of the patients who tried the procedure and remained hospitalized for one day or less; $30.09 \%(n=411)$, one to three days; $5.01 \%(n=85)$, four to seven days; $1.03 \%(\mathrm{n}=21)$, eight to eleven days; $12.12 \%$ ( $\mathrm{n}$ $=32)$, twelve to twenty-seven days and $0.4 \%(\mathrm{n}=4)$ were hospitalized for more than 28 days.

Regarding the counter-referral of patients with suicidal potential, $85.4 \%$ were not referred to any professional service,
$6.4 \%$ for psychiatry or private clinics, $5.3 \%$ for the and only $3 \%$ were referred for primary health care.

Table 2. Prevalence of suicide attempts related to hospitalization time and counterreference between the years 2009 to 2012

\begin{tabular}{lll}
\hline & $\mathbf{N}$ & $\mathbf{\%}$ \\
\hline Length of stay & 1,654 & 100 \\
Até $<1$ & 1,005 & 60.08 \\
01 a 03 & 411 & 30.09 \\
04 a 07 & 85 & 5.01 \\
08 a 11 & 21 & 1.03 \\
12 a 27 & 32 & 12.12 \\
$>28$ & 4 & 0.04 \\
Counter-referencing & & \\
Not forwarded & 1,412 & 85.4 \\
Primary attention & 50 & 3.0 \\
Private Psychiatric Clinic/Private Service & 105 & 6.4 \\
CAPS & 87 & 5.3 \\
\hline
\end{tabular}

Table 3 shows the distribution of suicide attempts associated with the medical diagnosis from the International Classification of Diseases (ICD-10). The study presented 3 prevalent ICD-10 codes, $40.2 \%(n=665)$ of suicide attempts diagnosed as ICD-10 X-68, which refers to self-poisoning and intentional exposure to pesticides; $39.2 \%(n=649)$ as ICD-10 $\mathrm{X}-61$, which represents intentional self-poisoning and exposure to antiepileptic, sedative-hypnotic, antiparkinsonian and psychotropic drugs not classified elsewhere such as antidepressants, barbiturates, neuroleptics, psychostimulants, and $8.8 \%$ of the population $(n=145)$ were diagnosed as ICD-10 $\mathrm{X}-64$ self-poisoning and intentional exposure to other drugs and unspecified biological substances.

Table 3. Frequency and percentage of suicide attempts associated with the diagnosis ICD-10, 2009 to 2012

\begin{tabular}{|c|c|c|}
\hline \multirow{2}{*}{ DIAGNOSIS ICD-10 } & \multirow{2}{*}{$\begin{array}{l}\mathrm{N} \\
1,654\end{array}$} & \multirow{2}{*}{ \% 100} \\
\hline & & \\
\hline X-68 Autointoxication by and exposure, intentional, to pesticides-residence. & 665 & 40.2 \\
\hline $\begin{array}{l}\text { X-61 Autointoxication by and exposure to intentional anticonvulsant drugs (antiepileptics), sedatives, hypnotics, antiparkinsonians and } \\
\text { psychotropic drugs not elsewhere classified. }\end{array}$ & 649 & 39.2 \\
\hline X-64 Autointoxication by and exposure, intentional, to other drugs and biological substances and ace unspecified residence. & 145 & 8.8 \\
\hline X-60 Autointoxication by and exposure, intentional, to analgesics, antipyretics and antirheumatic, non-opiates. & 72 & 4.4 \\
\hline X-49 Accidental poisoning (poisoning) from exposure to other and unspecified harmful chemicals. & 10 & 0.6 \\
\hline X-62 Autointoxication by and exposure to narcotics and psychodysleptics (hallucinogens) not classified elsewhere. & 10 & 0.6 \\
\hline \multicolumn{3}{|l|}{ X-40 Poisoning accidental intoxication by exposure to analgesics, antipyretics and antirheumatics, not opiates. } \\
\hline X-48 Accidental poisoning from exposure to pesticides. & 10 & 0.4 \\
\hline \multicolumn{3}{|l|}{ X-71 Intentional self-harm by drowning and submersion-residence. } \\
\hline X-63 Autointoxication by and intentional exposure to other pharmacological action substances on the autonomic nervous system. & 8 & 0.5 \\
\hline X-81 Intentional self-harm by precipitation or permanence in front of a moving object-residence. & 5 & 0.3 \\
\hline X-28 Contacts with other specified venomous plants-residence. & & \\
\hline $\begin{array}{l}\text { X-42 Poisoning accidental poisoning by exposure to narcotics and psychodysleptics (hallucinogens) not elsewhere classified. } \\
\text { X-43 Accidental poisoning (poisoning) by exposure to other pharmacological action substances on the autonomic nervous system. } \\
\text { X-70 Intentional self-harm by hanging, strangulation and suffocation - residence. }\end{array}$ & 4 & 0.3 \\
\hline
\end{tabular}


The less frequent diagnoses were: 0.1\%, ICD-10 X-28 (contact with other venomous plants specified); (Accidental poisoning/intoxication by exposure to other pharmacological action on the autonomic nervous system) and 0.1\% CID-10 X-70 (intentionally self-harm by hanging, suffocation).

After emergency treatment, $97 \%$ of the patients were discharged from hospital, these data related to the diagnosis ICD-10 X-68, shows that $40.2 \%$ used this method as a suicide attempt.

Table 4 presents the Sperman correlation of the variables, Time of hospitalization, Consequence of the attempt and the Care received from the cases of suicide attempt, where the statistical significance is relevant.

When Spearman Correlation Analysis was applied, it was observed that a positive correlation coefficient was observed, as a consequence of the attempt and the length of hospitalization (.289). Where the patients who were hospitalized for a longer time presented a greater risk to life. Another variable worth mentioning is Care and Time of hospitalization (.955), in which patients who attempted suicide and received medical care/health care also spent more time in hospital.

Table 4. Spearman Rô correlation of the variables: Time of hospitalization, consequence of attempt and care received

\begin{tabular}{lllll}
\hline Variables & Correlation & TH $^{*}$ & CA** $^{* *}$ & CR $^{* * *}$ \\
\hline TH & Correlation coefficient & 1.000 & .289 & .955 \\
CA & Correlation coefficient & .289 & 1.000 & .303 \\
CR & Correlation coefficient & .955 & .303 & 1.000 \\
\hline *Time of
\end{tabular}

\section{Discussion}

Suicide cases and attempts showed a significant increase. When related to exogenous intoxication, the number of suicides increases more, becoming this self-directed violence greater and more frequent. According to a study carried out in Rio de Janeiro, Brazil ${ }^{[7]}$ suicide attempts by hanging are among the main causes of suicide attempts. Then the suicide attempts by intoxication. These cases require investigation, because when not detected early, in addition to causing damage to oneself, they cause psychological damages to the relative

In Mesoregion North Paranaense in the years 2002 to 2011, most of the participants were intoxicated by pesticides, $67.12 \%$ were male, primarily reaching the age group 20-39 years. ${ }^{[8]}$ In this research most were intentionally intoxicated. The age range ranged from 17 to 75 years, with the mean age of 30 years, corroborating the study regarding the age group. In the data from another study ${ }^{[5]}$ the prevalence was female and the age group 15 to 29 years old. In the study conducted in Ilam province in Western Iran, the highest incidence rate and suicide attempts were observed between 15 and 24 years. ${ }^{[9]}$

A study in the city of Taubaté-SP identified that there was a predominance of incomplete grade 2 , this for the individual exposed to suicide attempt. Some medical records did not have the information in question, being represented by the item "not applicable" (15.5\%). As in the present study, many cases were not represented by the degree of schooling. In Rio de Janeiro, similar results showed that patients at the primary level suffered greater suicide attempts $(64=66.7 \%),{ }^{[5]}$ Compared with the Ilam study in western Iran, the author suggests that the higher an individual's level of education, the greater his level of knowledge, helping him to better analyze problems and overcome difficulties less emotional and more rational. Educational level, therefore, can be considered as a deterrent against suicide. ${ }^{[9]}$

A study of suicide attempts by exogenous intoxication in Ceará, Brazil ${ }^{[10]}$ found that it was higher among women (55\%), followed by $45 \%$ among men. In the present study, however, the highest number of suicide attempts was among men. It is not possible to affirm the real number of those who attempted suicide and of those who managed to carry out the action, since did not include this category. In Morocco the results confirm the data from the present study, where the highest number of suicides was performed by men (13.1\%) than by women $(8.1 \%)$. This confirms that the mortality risk for men is higher than for women, who are in higher risk of morbidity. ${ }^{[11]}$

The months before the end of the year were the months in which suicide attempts occurred most. A descriptive observational epidemiological study demonstrates a relationship with the present study: the months with the highest number of notifications were May (12.8\%) and October (11\%), followed by December and August, both with 10.3\%. ${ }^{[12]}$

It was possible to identify that the largest number of patients who tried intoxication received "emergency care followed by hospital discharge". This indicates, in the present study, that there were more attempts than effective suicide when compared to patients who were in more severe states and died. Perhaps, it is related as a way of "drawing attention", where in the study carried out by Psychologists, that suicide is socially denoted as a way of drawing attention, where the meaning of life ceases to exist, but must be perceived with a request for help from a person who is experiencing deep psychological suffering. ${ }^{[13]}$

In two studies were found data that present a comparison in relation to the type of care received/evolution of the case. 
In one from Ceará, Brazil ${ }^{[10]}$ approximately $72.8 \%$ of the patients were cured and had no sequelae. In the study carried out at the Poison Control Center of the Regional University Hospital of Maringá, Brazil (CCI / HUM) ${ }^{[14]} 96.3 \%$ evolved to cure. In Iran the length of hospital stay, to $52.6 \%$ was 2 days and the mortality rate was $0.4 \% .{ }^{[15]}$ The risk of death exists in all cases, however, in the studies surveyed, the risk was lower. The patients evolved to improvement and discharge from hospital, presenting no greater risk to themselves.

The variable consequences of suicide attempts and hospitalization time, when correlated, showed a Spearman correlation coefficient of $r=0.29$, indicating that $8.3 \%$ of the patients who received hospital care presented life risk $(p<$ $.01)$. As for the level of aggravation, it must be taken into account the amount of toxic products that was ingested and the age, since it may be related to the psychic suffering that this patient is experiencing.

An example is the group of elderly people who are more likely to be lethal because they have lower physical resistance, easy access to medications and chronic diseases, and are contributing factors to a slow recovery.

A retrospective review paper found that $28.7 \%$ of patients were referred to psychiatric institutions. ${ }^{[9]}$ A study in the city of Newcastle, Australia, ${ }^{[16]}$ found that, after assessing patients for the risk of suicide, $27.6 \%$ were not referred for admission to a psychiatric service, $45.3 \%$ did not receive a psychiatric evaluation and $29.2 \%$ of the individuals were referred for psychiatric hospitalization. In this respect, from the official databases, it is reiterated that there is a $61.3 \%$ probability of underreporting in cases of attempted suicide, thus suggesting the importance of better professional evaluation and recording of information. ${ }^{[7]}$

The same research cited above indicated that $90 \%$ of the patients who were hospitalized in the last 12 months had a suicidal plan or high levels of suicidal ideation, ${ }^{[7]}$ suggesting that the patients are cared for, but not referred to any service of the Network of Psychosocial Attention that has as a flow the host with consent and responsibility of the user based on the logic of Harm Reduction by the Unit of Reception Unit (UA), clinical follow up and psychosocial aspects of the user and family. ${ }^{[17]}$ These visits after hospitalization in the emergency room would minimize the risk of a new suicide attempt.

A paper ${ }^{[16]}$ on suicide prevention from pesticide control suggests that efficient monitoring of banned agrochemicals provided by the black market, which has poor quality control and labeling, should be carried out to reduce the number of

Published by Sciedu Press intoxications by pesticides and intentional death. In WHO data, ${ }^{[18]}$ Class I pesticides (organochlorines) are one of the major public health problems due to their chemical compositions that are highly detrimental to human health and the environment. There are five classes that have been banned in the US and Europe since 1985, Brazil since 1999 and China only two years ago. With the law the number of poisoning deaths in China has been reduced and has been associated with a possible $10 \%$ to $15 \%$ drop in overall suicide rates in women.

One study showed that $70.6 \%$ of the men were poisoned with pesticides, compared to the current study, $58.8 \%$ were male. ${ }^{[10]}$ The fact that man is in direct contact with the crop, preparation and administration, makes it easier to access the product. Another research ${ }^{[16]}$ indicates that $41 \%$ were also of men who committed the act with pesticides. In the Beijing study in China, located in the countryside, men are three times more likely to commit suicide than women. Rural suicide rates are about three times higher than in urban areas, increasing to almost five times in women aged 25-34. ${ }^{[19]}$

Pesticide ingestion in Asian countries is a common method of suicide. ${ }^{[20]}$ In Korea, a survey carried out with n=1301 patients shows that the highest number of suicide attempts was with the herbicide $(74.3 \%)$, followed by insecticide (13.8\%) and pesticide (7.9\%). ${ }^{[19]}$ The most frequent cases of suicide attempts were represented by the diagnosis ICD-10 X-68 (by intentional exposure to pesticides-residence). In Ceará, Brazil 10.17\% were from agrochemicals used in agriculture and only $3 \%$ were domestic pesticides. ${ }^{[10]}$

In Iran, there are other data: the most common agent was antidepressant poisoning (17.6\%), followed by analgesics $(12.8 \%)$ and other psychotropic drugs (6.1\%). Type of suicide attempt was more frequent in drug intoxications (69.3\%) and by sharp objects (17.1\%) or other methods (13.6\%). Men $(27.8 \%)$ are more likely to be cut than women $(11.5 \%)$, while women appear to be more likely to self-poison $(76.5 \%)$ men (49.5\%), with a $p$ value less than .001 . As for the type of drug used, benzodiazepines or barbiturates (47.9\%), multiple drugs $(22.4 \%)$ and non-psychiatric medications (15.10\%) were the most ingested drugs. These data suggest that the manner in which poisoning occurs can be diversified when compared to the individual's sex, region, culture, and country of residence. ${ }^{[21]}$

Suicide is a public health problem that requires a differentiated approach to its prevention from the multiprofessional team, aiming at its early detection. It was possible to observe that many data from the TIC were omitted or not found, and therefore need to be reviewed as to the way it was obtained, and it was also a limitation to the study, since it would al- 
low identification also, better, the relationship between the level of schooling and the suicide attempt, as well as other variables.

It is necessary, therefore, to invest in the training of professional health services to better identify the suicide attempt signs of improvement in information recording system as well as in their own mental health care network, aiming to avoid new suicide attempts.

\section{Conclusion}

It can be seen that suicide attempts were more frequent among men, where most of the population lived in the urban area. The form that the individual made this attempt was frequent with pesticides that had in their homes, which is remarkable, because they presented pesticides at home, since these patients resided in the urban zone. However, it was not possible to measure how many of these patients who used pesticides in their residence lived in the rural area and how many in the urban area, we only had this data in a general way.

One limitation of the study to be highlighted is that Information Recording System record did not include records that presented the outcome of these patients in relation to new suicide attempts.

It was also observed that the highest numbers of suicide attempts occurred in the months closest to the end of the year on festive dates, specifically at Christmas and New Year. However, it was not possible to find many studies that prove the relationship between suicide attempts and holiday dates. These dates are considered of great importance for most human beings, because it is a time of fellowship with the family, exchange of gifts, and feedback of memories, whether they are pleasant or not.

Health professionals need to be trained in order to identify and know how to intervene in cases of suicide attempts, each within its specificity and complexity. Another important fact is that many cases are not referred to any health service, when the majority of those cared for should have been referred to another health care facility.

It is also suggested that the risk factor for mental disorder should be included in the notification form, since this information places a greater risk of suicide on patients with suicidal ideation.

\section{CONFLICTS OF INTEREST Disclosure}

The authors declare that there is no conflict of interest.

\section{REFERENCES}

[1] World Health Organization (WHO). Preventing suicide: a global imperative. Geneva: WHO; 2014.

[2] Dantas JSS, Uchôa SL, Cavalcante TMC, et al. Profile of the patient with exogenous intoxication by "chumbinho" in the initial approach in emergency service. Rev. Eletr. Enferm. 2013; 15(1).

[3] Werneck GL, Hasselmann MH, Phebo LB, et al. Attempted suicide in a general hospital in Rio de Janeiro, Brazil. Cad. Public Health 2006; 22(10): 2201-6.

[4] Diehl A, Laranjeira R. Suicide attempts and substance use in an emergency room sample. J. Bras. Psiquiatr. 2009; 58(2): 86-91. https://doi.org/10.1590/S0047-20852009000200003

[5] Santos SA, Lovisi G, Legay L, et al. Prevalence of mental disorders in suicide attempts at an emergency hospital in Rio de Janeiro, Brazil. Cad. Saúde Pública. 2009; 25(9): 2064-74.

[6] World Health Organization (WHO). Suicide prevention (SUPRE): multisite intervention study on suicidal behaviours - SUPRE-MISS. WHO: Geneva, 2001.

[7] Santos AS, Legay LF, Aguiar FP, et al. Attempts and suicides due to exogenous intoxication in Rio de Janeiro, Brazil: analysis of information through probabilistic link. Cad. Public Health. 2014; 30(5).

[8] Neves PDM, Bellini M. Poisoning by agrochemicals in the north central region of Paraná, Brazil - 2002 to 2001. Ciênc. Saúde Col. 2013; 18(11): 3147-56.

[9] Azizpour Y, Asadollahi K, Sayehmiri K, et al. Epidemiological survey of intentional poisoning suicide during 1993-2013 in Ilam Province,
Iran. BMC Public Health. 2016; 16(1): 902. PMid:27576701 https : //doi.org/10.1186/s12889-016-3585-9

[10] Oliveira EN, Félix TA, Mendonça CBL, et al. Attempted suicide by exogenous intoxication: context of compulsory notifications. Revista Eletrônica Gestão \& Saúde. 2015; 6(3): 2497-11.

[11] Siham M, Abdelmajid S, Hind H, et al. Suicides par intoxication dans la région de Souss-Massa-Drâa au Maroc. Saúde Pública. 2013; 23(3): 343-350.

[12] Vieira LP, Santana VTP, Suchara EA. Characterization of suicide attempts by exogenous substances. Cad. Saúde Colet. 2015; 23(2): 118-123.

[13] Silva K, de FA, Alves MA, Couto PD do. Suicide: an existential choice in the face of human despair. Pretext - Journal of the Graduation in Psychology of PUC Minas. 2016; 1(2).

[14] Trevisan EPT, Santos JAT, Oliveir MLF. Attempted suicide of women: data from a toxicological assistance center in Paraná. REME. 2012; $17(2)$.

[15] Zohre E, Ayrik C, Bozkurt S, et al. Retrospective Analysis of Poisoning Cases Admitted to the Emergency Medicine. Arch Ir Med. 2015; 18(2): 117-22.

[16] Carter GL, Safranko I, Lewin TJ, et al. Psychiatric hospitalization after deliberate self-poisoning. Suicide Life Threat Behav. 2006; 36(2): 213-22. PMid:16704325 https://doi .org/10.1521/suli. 200 6.36 .2 .213

[17] Quinderé PHD, Jorge MSB, Franco TB. Network of Psychosocial Attention: the place of mental health. Physis Revista de Saúde Coletiva. 2014; 24(1): 253-271. 
[18] World Health Organization (WHO). ICD-10 International Statistical Classification of Diseases and Related Health Problems. São Paulo: Universidade de São Paulo. 2007.

[19] Zhang J, Jiang C, Jia S, et al. An Overview of Suicide Research in China. Arch Suicide Res. 2002; 6(2): 167-184. PMid:20686645 https://doi.org/10.1080/13811110208951174
[20] Lee JW, Hwang W, Kim JW, et al. Common Pesticides Used in Suicide Attempts Following the 2012 Paraquat Ban in Korea. J Korean Med Sci. 2015; 30(10): 1517-1521. PMid:26425052 https: //doi.org/10.3346/jkms.2015.30.10.1517

[21] Zeppegno P, Gramaglia C, Castello LM, et al. Suicide attempts and emergency room psychiatric consultation. BMC Psychiatry. 2015; 15(13). 